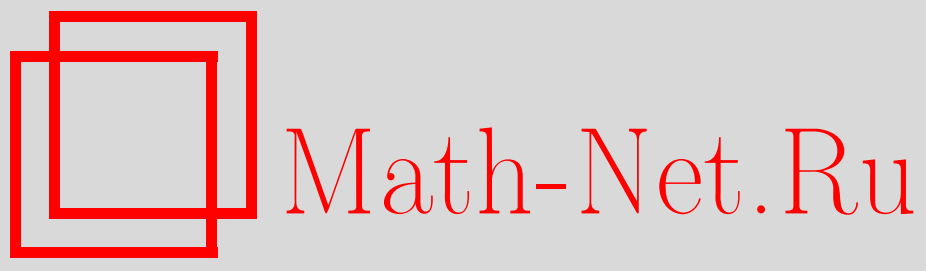

М. Спрефико, Об одном обобщении гамма-функции Эйлера, Функи. анализ и его прил., 2005, том 39, выпуск 2, 87-91

DOI: https://doi.org/10.4213/faa46

Использование Общероссийского математического портала MathNet.Ru подразумевает, что вы прочитали и согласны с пользовательским соглашением

http://www . mathnet.ru/rus/agreement

Параметры загрузки:

IP: 3.89 .197 .203

26 апреля 2023 г., $07: 14: 29$

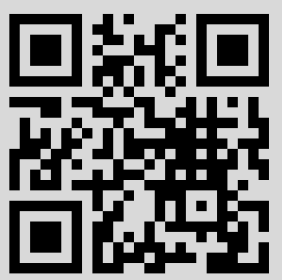




\section{Об одном обобщении гамма-функции Эйлера}

(C) 2005. М. СПРЕФИКО

1. Введение. Рассмотрим регуляризованное произведение Вейерштрасса

$$
F_{\beta}(z)=\prod_{n=1}^{\infty}\left(1+\frac{z}{n^{\beta}}\right) e^{\sum_{j=1}^{p} \frac{(-1)^{j}}{j} \frac{z^{j}}{n^{j \beta}}},
$$

где $\beta$ вещественно и положительно, $p=[1 / \beta]$ и $\gamma_{\beta}=-\ln F_{\beta}(1)$. Определим функцию $\Gamma_{\beta}(z)$ формулой $1 / \Gamma_{\beta}(z)=z e^{\gamma_{\beta} z} F_{\beta}(z)$. Это аналитическая функция переменной $z$ во всей комплексной плоскости, за исключением точек $z=$ $0,-1^{\beta},-2^{\beta}, \ldots$, в которых она имеет простые полюсы, причем $\Gamma_{\beta}(1)=1$. В этой заметке мы анонсируем основные свойства функции $\Gamma_{\beta}$, а подробности и полные доказательства будут опубликованы в другой работе. Поскольку $\Gamma_{1}(z)=\Gamma(z)$, то функция $\Gamma_{\beta}$ представляет собой естественное обобщение функции Эйлера, и мы показываем, что все свойства, характеризующие гамма-функцию и не зависящие от «линейности» членов определяющей последовательности (см. разд. 2), выполнены весьма естественным и простым образом и для $\Gamma_{\beta}$. В частности, мы выписываем функциональное уравнение, ряд Тейлора, а также асимптотическое разложение при больших $z$. Примечательно, что последнее мы получаем, доказывая существование полного асимптотического разложения соответствующей функции теплопроводности (лемма 3). Последняя имеет вид $\sum_{n=1}^{\infty} e^{-a_{n} t}$ для некоторой последовательности $a_{n}$, и в общем случае весьма мало шансов получить для таких функций полное разложение. Труден уже случай двумерной сферы [4]. При $\beta>1$ обобщение формулы Әйлера для гамма-функции задается выражением

$$
F_{\beta}(z)=\lim _{m \rightarrow \infty} \frac{\left(1^{\beta}+z\right)\left(2^{\beta}+z\right) \cdots\left(m^{\beta}+z\right)}{1^{\beta} 2^{\beta} \cdots m^{\beta}},
$$

которое продолжается в область $\beta<1$ с подходящими регуляризующими множителями. Эта формула показывает, что ни функциональное уравнение, ни интегральное представление гамма-функции, вероятно, не обобщаются на случай функции $\Gamma_{\beta}$, поскольку они основаны на «линейности» общего члена.

Помимо абстрактного интереса $[6,8]$, изучение такого рода функций мотивировано тем, что они возникают при рассмотрении регуляризованного определителя $[5,9]$ вещественных степеней оператора Лапласа на окружности ${ }^{1)}$ [3]: определитель $\operatorname{det}_{\zeta}\left(-\Delta_{S^{1}}^{\beta}+a\right)$ равен $\Gamma_{\beta}(a)$, что обобщает формулу Лерха (лемма 3$)$. В частности, асимптотики логарифма $\ln \Gamma_{\beta}$ задают поведение при малом или большом массовом члене.

2. Спектральные функции. Рассмотрим последовательность $S_{\beta, a}=\left\{a_{n}=\right.$ $\left.n^{\beta}+a\right\}$, где $\beta \neq 1$ фиксировано ${ }^{2)}$. Последовательность $S_{\beta, a}$ имеет конечный

1) В стандартном случае $F_{2}(z)=\frac{\operatorname{sh} \pi \sqrt{z}}{\pi \sqrt{z}}$.

2) Заметим, что при $\beta=1$ общий член однородной последовательности $S_{1,0}$ линеен по переменной $n$. В действительности это свойство является причиной некоторых основных свойств гамма-функции, и именно поэтому последние не наблюдаются в общем нелинейном случае $\beta \neq 1$. 
показатель сходимости $1 / \beta$ и единственную точку накопления на бесконечности. Мы сопоставим последовательности $S_{\beta, a}$ следующие спектральные функции: дзета-функцию $\zeta_{\beta}(s, a)=\sum_{n=1}^{\infty}\left(n^{\beta}+a\right)^{-s}$, регуляризованное произведение Вейерштрасса $F_{\beta}(z)$, которое является целой функцией порядка $1 / \beta$ и рода $p=[1 / \beta]$, функцию теплопроводности $f_{\beta}(t, a)=\sum_{n=0}^{\infty} e^{-\left(n^{\beta}+a\right) t}$ и резольвентную функцию, определенную формулой $R_{\beta}(\lambda, a)=\sum_{n=1}^{\infty} \frac{1}{\lambda-n^{\beta}-a}=$ $-\zeta_{\beta}(1, a-\lambda)$ при $\beta>1$ и $\lambda$, лежащем в произвольном секторе, не содержащем чисел $a_{n}$. Для произвольного $\beta$ мы можем ввести только производные $R_{\beta}^{(p)}(\lambda, a)=(-1)^{p} \sum_{n=1}^{\infty}\left(\lambda-n^{\beta}-a\right)^{-p-1}=-\zeta_{\beta}(p+1, a-\lambda)$.

Используя теорему Плана таким же образом, как и в [7], мы получаем полное асимптотическое разложение функции теплопроводности. Пусть $\beta=[\beta]+(\beta)$.

ЛЕмма 1. При $t \rightarrow 0^{+}$справедливо разложение $f_{\beta}(t, 0) \sim \Gamma\left(1+\frac{1}{\beta}\right) t^{-1 / \beta}+$ $\frac{1}{2}+\sum_{m=1}^{\infty} \frac{(-1)^{m}}{m !} c_{m} t^{m}$, гдe

$$
\begin{aligned}
c_{2 m} & =2 \sum_{j=0}^{m-1}(-1)^{j+1}\left(\begin{array}{c}
2 m \\
2 j+1
\end{array}\right) \cos ^{2 j+1} \frac{\pi}{2}(\beta) \sin ^{2 m-2 j-1} \frac{\pi}{2}(\beta) \int_{0}^{\infty} \frac{y^{2 m \beta}}{e^{2 \pi y}-1} d y, \\
c_{2 m+1} & =2 \sum_{j=0}^{m}(-1)^{m+j+1}\left(\begin{array}{c}
2 m+1 \\
2 j
\end{array}\right) \cos ^{2 j} \frac{\pi}{2}(\beta) \sin ^{2 m+1-2 j} \frac{\pi}{2}(\beta) \int_{0}^{\infty} \frac{y^{(2 m+1) \beta}}{e^{2 \pi y}-1} d y .
\end{aligned}
$$

3. Дзета-функция. Основные свойства обобщенной дзета-функции Гурвица $\zeta_{\beta}(s, a)$ можно получить стандартными методами [2], но мы дадим простое доказательство взаимосвязи основных дзета-инвариантов с произведениями Вейерштрасса [9].

ЛЕмма 2. Функиия $\zeta_{\beta}(s, a)$ обладает регулярным аналитическим продолжсением на всю комплексную плоскость, за исключением простых полюсов в точках $s=\frac{1}{\beta}-j$ при всех $j=0,1,2, \ldots$, если $\frac{1}{\beta} \notin \mathbb{N}$, или при $0 \leqslant j \leqslant \frac{1}{\beta}-1$, если $\frac{1}{\beta} \in \mathbb{N}_{0,1}$. При этом

$$
\operatorname{Res}_{1}\left(\zeta_{\beta}(s, a), s=\frac{1}{\beta}-j\right)=\frac{(-1)^{j}}{j !} \frac{\Gamma\left(1+\frac{1}{\beta}\right)}{\Gamma\left(\frac{1}{\beta}-j\right)} a^{j} .
$$

СлЕДСТВИЕ 1. Для коэффициентов в разложении функиии теплопроводности справедлива альтернативная бормула $c_{m}=\zeta_{\beta}(-m, 0)=\zeta(-\beta m)$.

ЛЕмма 3. Справедливы формуль

$$
\begin{aligned}
\zeta_{\beta}(0, a)= \begin{cases}-\frac{1}{2}, & \frac{1}{\beta} \notin \mathbb{N}, \\
-\frac{1}{2}+(-1)^{1 / \beta} a^{1 / \beta}, & \frac{1}{\beta} \in \mathbb{N}_{0,1},\end{cases} \\
\zeta_{\beta}^{\prime}(0, a)=-\frac{\beta}{2} \ln 2 \pi+\sum_{j=1}^{p-1} \frac{(-1)^{j}}{j} \zeta(\beta j) a^{j}-\ln F_{\beta}(a) \\
+ \begin{cases}\frac{(-1)^{p}}{p} \zeta(\beta p) a^{p}, & \frac{1}{\beta} \notin \mathbb{N}, p \neq 0 \\
(-1)^{1 / \beta}[(\beta+1) \gamma+\psi(1 / \beta)] a^{1 / \beta}, & \frac{1}{\beta} \in \mathbb{N}_{0,1} .\end{cases}
\end{aligned}
$$


ДокАЗАтельство. При $|a|<1$ разложим степени двучлена в определении дзета-функции и получим $\zeta_{\beta}(s, a)=\sum_{j=0}^{\infty}\left(\begin{array}{c}-s \\ j\end{array}\right) \zeta_{\beta}(s+j, 0) a^{j}$, где $\zeta_{\beta}(s, 0)=\zeta(\beta s)$. Если $1 / \beta \notin \mathbb{N}$, то мы можем вычислить и ряд, и его производную по $s$ при $s=0$. При $1 / \beta \in \mathbb{N}_{0,1}$ трудности связаны с $p$-м членом, который можно вычислить, используя разложения при $s=0$ :

$$
\left(\begin{array}{c}
-s \\
j
\end{array}\right) \zeta_{\beta}(s+j, 0)=\frac{(-1)^{j}}{j}\left\{R_{1}(j)+\left[R_{0}(j)+(\gamma+\psi(j)) R_{1}(j)\right] s\right\}+O\left(s^{2}\right),
$$

где

$$
R_{k}(j)=\operatorname{Res}_{k}\left(\zeta_{\beta}(s, 0), s=j\right)=\left\{\begin{array}{ll}
\gamma, & k=0 \\
1 / \beta, & k=1
\end{array} .\right.
$$

Пользуясь леммой 3, мы обобщаем лемму о факторизации Чои и Куина [1]: СлЕдСтвиЕ 2. Справедливы формулъь

$$
\begin{aligned}
& \zeta_{2 \beta}\left(0, a^{2}\right)=\frac{1}{2}\left[\zeta_{\beta}(0, i a)+\zeta_{\beta}(0,-i a)\right], \\
& \zeta_{2 \beta}^{\prime}\left(0, a^{2}\right)=\zeta_{\beta}^{\prime}(0, i a)+\zeta_{\beta}^{\prime}(0,-i a)- \begin{cases}2(-1)^{p / 2} \sum_{j=1}^{p / 2} \frac{1}{2 j-1} a^{p}, & \text { если } p \text { четно, } \\
0, & \text { если } р \text { нечетно. }\end{cases}
\end{aligned}
$$

4. Свойства гамма-функции. Наши первые результаты следуют из определения ${ }^{1)}$.

ПРЕДЛОЖЕНИЕ 1. Справедлива формула $\Gamma_{\beta}(i z) \Gamma_{\beta}(-i z)=e^{\gamma_{2 \beta} z^{2}} \Gamma_{2 \beta}\left(z^{2}\right)$.

ПРЕДЛОЖЕНИЕ 2. Если $\beta>1$, mo

$$
\left(1-\frac{x}{z}\right) e^{-\gamma_{\beta} x} \prod_{n=1}^{\infty}\left(1-\frac{x}{n^{\beta}+z}\right) e^{x / n^{\beta}}=\frac{\Gamma_{\beta}(z)}{\Gamma_{\beta}(z-x)} .
$$

В качестве следствия можно вычислить общий класс бесконечных произведений вида $\Pi=\prod_{n=1}^{\infty} P\left(n^{\beta}\right) / Q\left(n^{\beta}\right)$, где $P(x)$ и $Q(x)$ - два многочлена одной и той же степени $J$, а $Q$ не имеет корней вида $n^{\beta}$. Непосредственные вычисления дают

$$
\Pi=\prod_{j=1}^{J} \frac{b_{j} \Gamma_{\beta}\left(-b_{j}\right)}{a_{j} \Gamma_{\beta}\left(-a_{j}\right)} e^{\gamma_{\beta}\left(b_{j}-a_{j}\right)},
$$

где $a_{j}$ и $b_{j}$ - корни многочленов $P$ и $Q$ соответственно.

Ряд Тейлора функции $\ln \Gamma_{\beta}$ можно получить также из леммы 3 . В качестве альтернативного варианта можно использовать регуляризованную резольвентную функцию

$$
\widetilde{R}_{\beta}(-z, 0)=\sum_{n=1}^{\infty}\left[\frac{1}{-z-n^{\beta}}+\sum_{j=0}^{p-1} \frac{(-z)^{j}}{n^{(j+1) \beta}}\right]
$$

что будет существенно в доказательстве предложения 4. Указанный выше ряд равномерно сходится в любой ограниченной области вместе с первыми $p$ производными, так что мы можем продифференцировать и при подходящей нормализации получить $\left(d^{k} / d z^{k}\right) \ln F_{\beta}(z)=(-1)^{k}(k-1) ! \widetilde{R}_{\beta}^{(k-1)}(-z, 0)$. Переходя к пределу, имеем $\widetilde{R}_{\beta}^{(k)}(-z, 0)=0$ при $0 \leqslant k \leqslant p-1$. Таким образом, доказано следующее утверждение.

1) Предложение 1 также может быть получено из следствия 2. 
ПреДЛОЖЕНИЕ 3. При малъх z справедлива формула

$$
\ln \Gamma_{\beta}(z)=-\ln z-\gamma_{\beta} z+\sum_{j=p+1}^{\infty} \frac{(-1)^{j}}{j} \zeta(\beta j) z^{j} .
$$

Наш последний результат - асимптотическое разложение при больших значениях аргумента. Доказательство состоит из двух шагов. Вначале, как и в доказательстве леммы 2, используется преобразование Меллина, чтобы выразить $p$-резольвентную функцию $R_{\beta}^{(p)}(\lambda, 0)$ как преобразование Лапласа функции теплопроводности; таким образом, асимптотическое разложение функции $R_{\beta}^{(p)}(\lambda, 0)$ задается подстановкой, использующей доставляемое леммой 1 разложение функции теплопроводности. Поскольку асимптотические разложения можно интегрировать, из указанного выше разложения мы получаем разложение логарифма произведения Вейерштрасса с точностью до конечного числа постоянных $b_{j}$. Здесь требуется некоторая осторожность при обращении с возможным слагаемым $1 / z$. Следует различать два случая в зависимости от того, выполнено включение $1 / \beta \in \mathbb{N}_{0,1}$ или нет ${ }^{1)}$. Вторым шагом является нахождение пока что неизвестных постоянных $b_{j}$. Для этого нам потребуется ввести следующее аналитическое представление дзета-функции:

$$
\zeta_{\beta}(s, 0)=\frac{1}{\Gamma(s)} \int_{0}^{\infty} t^{s-1} \frac{1}{2 \pi i} \int_{\Lambda_{c, \theta}} e^{-\lambda t} \widetilde{R}_{\beta}(\lambda, 0) d \lambda d t,
$$

где $\Lambda_{c, \theta}=\{\lambda \in \mathbb{C}:|\arg (\lambda-c)|=\theta\}$ для некоторых вещественных $c<1$ и $0<\theta<\pi$ превращается в контур ганкелевского типа. Интегрируя по частям сначала по $\lambda$, а затем по $t$, мы получаем $\ln F_{\beta}$ вместо регуляризованной резольвенты и дополнительный множитель $s$. Присутствие последнего обеспечивает нуль второго порядка при $s=0$ и позволяет применить стандартную технику $^{2)}$ (см., например, [2]) и известное разложение логарифма $\ln F_{\beta}$, чтобы найти вычеты при $s=0$. Сравнивая их со значениями дзета-функции Римана и ее производных, находим постоянные $b_{j}$. Мы получаем следующее утверждение ${ }^{3)}$.

ПредложенИЕ 4. Для больших $z$ nри $\arg (z) \neq 0$ справедлива формула

$$
\begin{aligned}
& \ln \Gamma_{\beta}(z) \sim-\frac{1}{2} \ln z+\frac{\beta}{2} \ln 2 \pi-\sum_{m=1}^{\infty} \frac{(-1)^{m}}{m} \zeta(-\beta m) z^{-m}-\gamma_{\beta} z \\
& \quad+ \begin{cases}\sum_{j=1}^{p} \frac{(-1)^{j+1}}{j} \zeta(\beta j) z^{j}+\frac{(-1)^{p+1} \pi}{\sin \pi(1 / \beta-p)} z^{1 / \beta}, & \text { если } \frac{1}{\beta} \notin \mathbb{N}, p=\left[\frac{1}{\beta}\right] \neq 0, \\
-\frac{\pi}{\sin (\pi / \beta)} z^{1 / \beta}, & \text { если } \frac{1}{\beta} \notin \mathbb{N}, p=\left[\frac{1}{\beta}\right]=0, \\
\sum_{j=1}^{p-1} \frac{(-1)^{j+1}}{j} \zeta(\beta j) z^{j}+(-1)^{p+1} z^{p}[\ln z+\gamma \beta], & \text { если } \frac{1}{\beta}=p \in \mathbb{N}_{0,1} .\end{cases}
\end{aligned}
$$

1) Заметим, что в первом случае обязательно $\beta<1$, так что второй случай содержит случай $\beta>1, p=0$. Заметим также, что $1 / \beta$ всегда отлично тот нуля, поскольку $\beta$ предполагается конечным.

2) При модификации контура нужна некоторая осторожность.

3) Отметим, что последняя формула покрывает и случай $p=1$. 
В заключение отметим, что постоянная $\gamma_{\beta}$ представляет собой естественное обобщение постоянной Эйлера $\gamma=\gamma_{1}$. Это можно увидеть, явно выписывая определяющий ряд или замечая, что $\gamma_{\beta}=\sum_{k=p+1}^{\infty} \frac{(-1)^{k}}{k} \zeta(\beta k)$. Из изложенных выше результатов следует также, что

$$
\gamma_{\beta}=\lim _{z \rightarrow 0} \frac{d}{d z} \ln z \Gamma_{\beta}(z)=\lim _{z \rightarrow 0}\left(\widetilde{R}_{\beta}(-z, 0)-\frac{1}{z}\right),
$$

в согласии с классическим случаем и независимо от функционального уравнения. С другой стороны, для произвольного $\beta$ уже неверно, что постоянная $\gamma_{\beta}$ совпадает с неособой частью соответствующей дзета-функции $\zeta_{\beta}(s, 0)$ при $s=1$.

\title{
ЛИтерАТУРА
}

1. Choi J., Quine J. R. Rocky Mountain J. Math., 26, 719-729 (1996). 2. Gilkey P. B. Invariance theorems, the heat equation, and the Atiyah-Singer index theorem. Studies in Adv. Math. CRC Press, 1995. 3. Morpurgo C. Duke Math. J., 114, 477-573 (2002). 4. Mulholland H. P. Proc. Cambridge Phil. Soc., 24, 280-289 (1928). 5. Sarnak P. Comm. Math. Phys., 110, 113-120 (1987). 6. Schuster R. Z. Anal. Anwendungen, 11, 229-236 (1992). 7. Spreafico M. Rocky Mountain J. Math., 33, 1499-1512 (2003). 8. Vardi I. SIAM J. Math. Anal., 19, 493-507 (1988). 9. Voros A. Comm. Math. Phys., 110, 439-465 (1987).

ICMC, Universidade de São Paulo, CEP 13560-970 São Carols, Brazil e-mail: mauros@icmc.usp.br
Поступило в редакцию 23 апреля 2003 г.

\section{Некоторые соотношения на двойные числа Гурвица*}

\author{
(c) 2005. С. В. ШАДРин
}

1. В этой заметке мы формулируем некоторые новые соотношения на двойные числа Гурвица. Эти соотношения приходят из теории пересечений на пространстве модулей кривых и являются прямым обобщением результатов, анонсированных в [3] и доказанных в [4].

Числа Гурвица, которые мы здесь изучаем, - это основной объект исследования в работе [2]. Мы рекомендуем работу [2], в частности, как хороший обзор по двойным числам Гурвица, содержащий наиболее полный набор ссылок по данной тематике.

2. Напомним определение чисел Гурвица. Зафиксируем целые числа $g \geqslant 0$, $n \geqslant 1$ и два неупорядоченных разбиения числа $n$ на целые положительные слагаемые, $n=a_{1}+\cdots+a_{p}=b_{1}+\cdots+b_{q}$. Рассмотрим пространство $\mathbb{C P}^{1} \mathrm{c}$ отмеченными точками $0, \infty$ и еще $2 g+p+q-2$ точками $z_{1}, \ldots, z_{2 g+p+q-2}$.

Множество мероморфных функций $f: C \rightarrow \mathbb{C P}^{1}$ степени $n$, определенных на кривых $C$ рода $g$ и таких, что $(f)=-\sum_{i=1}^{p} a_{i} x_{i}+\sum_{j=1}^{q} b_{j} y_{j}\left(x_{i}, y_{j}-\right.$

*Работа частично поддержана грантами РФФИ-01-01-00660, РФФИ-02-01-22004 и НШ1972.2002.1. 